\title{
Aplicando Design Thinking en una asignatura de FADE
}

\section{Blanca de-Miguel-Molina ${ }^{a}$, María de-Miguel-Molina ${ }^{b}$, Virginia Santamarina-Campos ${ }^{c}$ y Marival Segarra-Oña ${ }^{d}$}

${ }^{a}$ Universitat Politècnica de València, Departamento de Organización de Empresas (bdemigu@omp.upv.es), bUniversitat Politècnica de València, Departamento de Organización de Empresas (mademi@omp.upv.es), 'CUniversitat Politècnica de Valencia, Departamento de Conservación y Restauración de Bienes Culturales (virsanca@upv.es) y dUniversitat Politècnica de València, Departamento de Organización de Empresas (maseo@omp.upv.es).

\begin{abstract}
In this document we present how Design Thinking is applied in a degree not related to design, concretely in the degree of Business Administration and Management. This methodology is offered by the world's leading business schools. Through different tasks, students apply the method to review specific services, combining tasks in the classroom and outside it. In this document we explain the objectives of the course in which this method is applied, as well as the different tasks that have been elaborated to apply it. Finally, we include some results obtained in the subject as well as some conclusions from our experience applying Design Thinking.
\end{abstract}

Keywords: Design Thinking, services, service design, Business Administration and Management.

\section{Resumen}

En esta comunicación presentamos cómo se aplica el método de Design Thinking en una asignatura de un grado no relacionado con el diseño, en concreto en el grado de Administración y Dirección de Empresas. Esta metodología forma parte actualmente de la oferta formativa de las principales escuelas de negocio. A través de diferentes tareas, los alumnos aplican el método para revisar servicios concretos, combinando tareas en el aula y fuera de ella. En esta comunicación explicamos los objetivos de la asignatura en los temas en que se aplica este método, asi como las diferentes tareas que se han ido elaborando a lo largo de los cursos. Por último, incluimos algunos resultados obtenidos en la asignatura asi como algunas conclusiones de la experiencia obtenida en la aplicación del Design Thinking.

Palabras clave: Design Thinking, servicios, diseño de servicios, Administración y Dirección de Empresas. 


\section{Introducción}

Design Thing (DT en adelante) es un método que utiliza la manera de trabajar de los diseñadores para crear productos y servicios que se adapten a las necesidades de los usuarios (Brown, 2008). Su uso se ha extendido, en los últimos años, entre las empresas de servicios (Jaaron y Backhouse, 2018). Igualmente, las mejores escuelas de negocios han incluido el DT en su oferta formativa, en los campos de la creatividad e innovación (Zidulka y Kajzer-Mitchell, 2018) y el marketing (Chen et al., 2018). DT se enseña tanto en títulos de máster como de grado (Armstrong, 2016).

Entre las razones que explican su éxito se pueden citar, por ejemplo, que es un método que favorece la participación, el diálogo y el aprendizaje (Liedtka, 2018). Otros autores han señalado que educar en este método dará a los futuros gestores las herramientas necesarias para dirigir en entorno turbulentos, que destacan por los cambios en las tecnologías, los hábitos de consumo y los modelos de negocio (Schumacher y Mayer, 2018). Asimismo, el método de DT se considera un medio de soporte al aprendizaje experiencial (Zidulka y Kajzer-Mitchell, 2018).

En esta comunicación presentamos las tareas diseñadas para utilizar DT en una asignatura de la Facultad de Administración y Dirección de Empresas, durante los últimos tres cursos. La asignatura, con título Diseño de Servicios, de la idea a la implementación, se ofreció como optativa en el año 2015 a la Facultad de ADE, empezando a impartirse en el curso 2016-2017. Las tareas se han ido adaptando para mejorar el aprendizaje del alumno. Conforme su uso se ha ido extendiendo, el material disponible sobre casos de aplicación también ha aumentado. Asimismo, nuestra propia experiencia en la aplicación de DT en proyectos nos ha mostrado cuáles son las principales dificultades pero también las amplias posibilidades que permite este método (de-Miguel-Molina et al., 2018; SantamarinaCampos et al., 2018). Entre estas ventajas está que permite adaptarlo a cada situación concreta (Lewrick et al., 2018).

Es importante matizar que cuando pedimos a los alumnos de un grado de ADE que utilicen DT, les estamos pidiendo que salgan de su zona de confort. De trabajar con números y metodologías cuantitativas, les pedimos que se fijen en las palabras, las imágenes y que utilicen metodología cualitativa. Y, además, que salgan fuera del aula a observar y enfatizar con los usuarios del servicio que van a revisar.

La comunicación se estructura en otros cuatro apartados, además de esta introducción. En el segundo definimos los objetivos perseguidos en la asignatura al aplicar DT. A continuación, presentamos las diferentes tareas realizadas para su aplicación. Después incluimos algunos de los resultados obtenidos en la asignatura. Por último, exponemos algunas conclusiones y limitaciones en la aplicación de DT al caso concreto que explicamos. 


\section{Objetivos}

El objetivo principal de esta comunicación es presentar las tareas relacionadas con la aplicación práctica de la metodología de DT en una asignatura de la Facultad de Administración y Dirección de Empresas (UPV) durante los últimos tres cursos. Asimismo, la comunicación está enmarcada dentro del Equipo de Innovación y Mejora Educativa (EICE) "El proceso de enseñanza y aprendizaje fuera de las aulas, baños de realidad", en el que participan profesores de diferentes centros de la UPV con los que trabajamos estas dinámicas.

Los objetivos al aplicar la metodología de DT en la asignatura son:

- Que los alumnos trabajen en base a la cultura de servicio centrada en el usuario.

- Que utilicen el método de DT para revisar un servicio y plantear mejoras en él.

- Que dichas mejoras estén basadas en el viaje del cliente (customer journey).

- Que apliquen metodología cualitativa (entrevista cualitativa) para obtener información directamente del cliente y que observen su comportamiento.

- Que utilicen información en redes sociales que los clientes hayan escrito sobre el servicio que se revisa, si está disponible, para que la información proceda de diversas fuentes.

\section{Desarrollo de la innovación}

La explicación del método y la realización de diferentes tareas relacionadas con su aplicación se estructuran a través de los pasos que explicamos a continuación. Las primeras tareas tienen por objetivo aplicar en grupo y de manera organizada lo que necesitarán para la tarea evaluable, basada en aplicar DT, para la que dispondrán de más tiempo y será más extensa.

El primer paso consiste en la explicación de qué es el DT y cuáles son las fases del método. Para ello, se utiliza un caso sobre cómo IDEO lo aplica en servicios concretos. Con este caso se lleva a cabo la primera tarea (Figura 1), que se realiza en grupo en el aula. En dicha tarea se pide a los grupos que den una definición de qué es el DT, definan cuáles son las fases y qué herramientas se utilizan en cada una. Toda esta información está recogida en el caso, a través de ejemplos en los que IDEO aplicó el DT para diseñar servicios. Cuando terminan la tarea, se comparten las ideas y se formulan preguntas en base a los ejemplos del caso, para comprobar que han entendido en qué consiste. Utilizando la Figura 2, se hace un resumen de las fases y se conecta con la tarea 2 y la última tarea, que será la que evaluará los conocimientos aprendidos. 


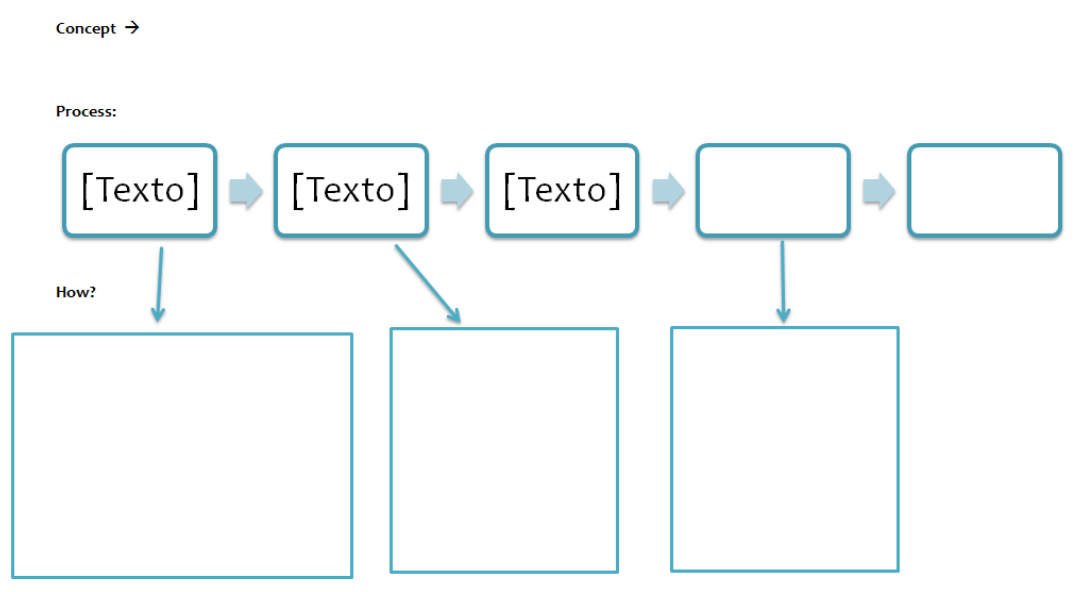

Fig. 1 Primera tarea sobre Design Thinking: concepto y fases

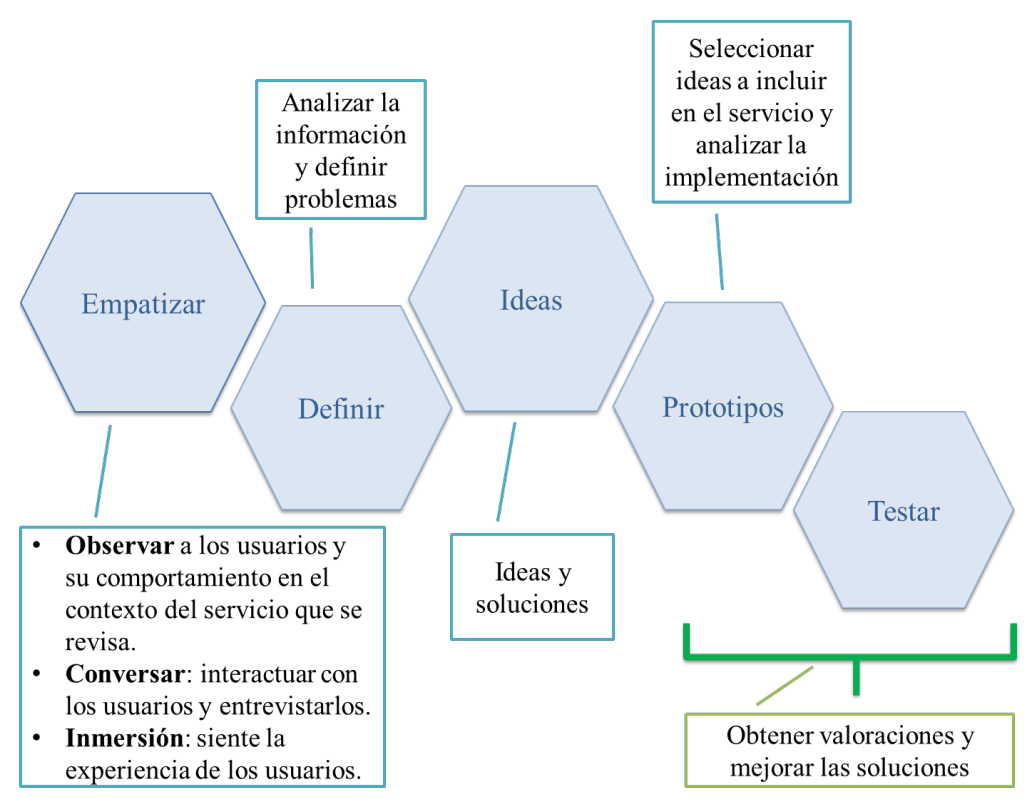

Fig. 2 Proceso de Design Thinking (d.school at Stanford University, 2018)

Una vez que conocen el método, se les explica en qué consiste la tarea 2. Esta tarea se centra en la Biblioteca de la UPV, a la que tendrán que ir para recoger la información necesaria para la siguiente sesión. El objetivo de esta tarea es que apliquen DT a la revisión del diseño de este servicio y, así, vayan practicando lo que necesitarán para la última tarea, una de las que se incluyen en la evaluación de la asignatura. Para llevar a cabo la tarea 2 se les dan las siguientes instrucciones:

- El diseño del servicio a revisar es la Biblioteca de la UPV.

- Tareas a realizar antes de la siguiente sesión: 
- Necesitareis preparar 5 preguntas, entre las que podeis incluir alguna de las siguientes:

- ¿Por qué vienes a la biblioteca? (si la respuesta es a estudiar, pregunta por qué estudiar en la biblioteca).

- ¿Qué espacios de la biblioteca utilizas más? (pregunta por un aspecto positivo y otro negativo en relación al espacio).

- ¿Qué servicio de la biblioteca utilizas más? (pregunta por un aspecto positivo y otro negativo en relación a ese servicio).

- ¿Hay algo que te gustaría mejorar?

El objetivo es que vean la diferencia entre preguntas en una encuesta y preguntas abiertas en una entrevista cualitativa, formato que deberán utilizar en la tarea final.

- Ve a la biblioteca y observa qué estacios se utilizan más. El objetivo es que incluyan la observación como fuente de información adicional.

- Selecciona una persona (una cada uno de vosotros) y hazle las preguntas que has preparado.

- Tareas a realizar en el aula:

- Organizad la información que habéis obtenido, por temas, viendo aspectos positivos y negativos (problemas) en cada caso.

- Seleccionad los problemas que consideréis más importantes y haced un brainstorming, dando ideas para solucionarlos.

- Piensa de manera crítica en las soluciones aportadas.

- Selecciona dos soluciones (aquellas que tendrían menor coste de implementación o crearían mayor valor para el usuario).

- Piensa en los potenciales problemas de su implementación y cómo resolverlos.

Para compartir la información y organizarla, se prepara un esquema (ver Figura 3) que les servirá de ayuda para incluir las ideas más importantes. Una vez rellenan la plantilla, los grupos comparten, de dos en dos, lo que han incluido en ella. Se les pide que seleccionen 23 problemas y soluciones. Por último, los grupos escriben en la pizarra estos últimos problemas y las soluciones, de manera que cada uno sólo añade problemas y soluciones no incluídos por el grupo anterior. El último paso consiste en preguntarles por posibles problemas en la implementación de cada solución propuesta y cómo se resolverían. 


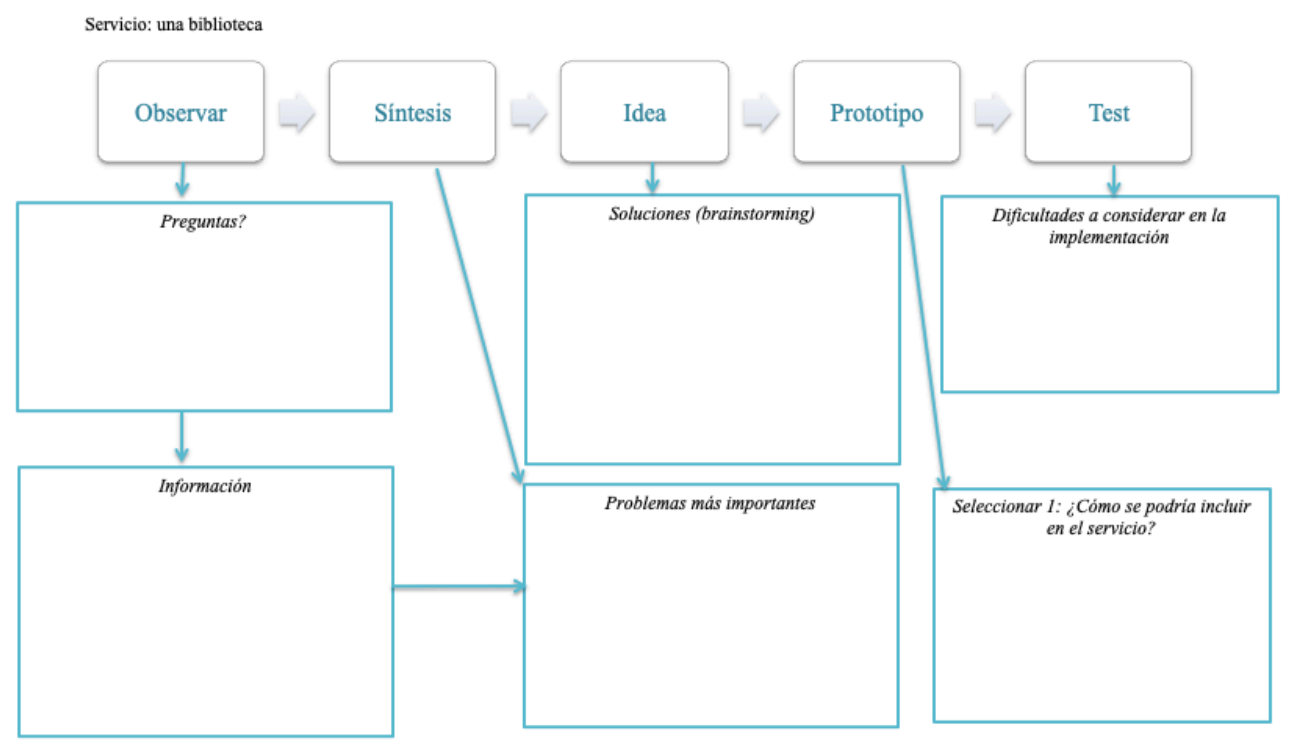

Fig. 3 Segunda tarea sobre Design Thinking: revisando el diseño del servicio de la biblioteca

Una vez finalizada la tarea 2, se explica en qué consistirá la tarea final, así como la diferencia respecto a las que han realizado hasta el momento. Se hace hincapié, especialmente, en que en la tarea de revisión del diseño del servicio de la biblioteca no se tuvo en cuenta el "viaje del cliente" (customer journey) para elaborar las preguntas de la entrevista. Sin embargo, la tarea final tendrá como eje central este viaje, que tendrán que representar primero. De ahí que el siguiente tema de la asignatura se dedique al viaje del cliente.

Por otra parte, no olvidamos que actualmente una parte importante de las opiniones de los clientes se encuentran en las redes sociales y webs específicas. Por ello, se dedica una sesión a trabajar sobre cómo obtener y analizar esta información, con un caso concreto y con una tarea que les permita aplicar el conocimiento adquirido en la tarea final. Los alumnos realizan una tarea que combina la información de la web Trypadvisor sobre un hotel con el viaje del cliente.

La tarea final es una de las dos tareas con más peso, individualmente, en la evaluación de la asignatura. A grandes rasgos, consiste en revisar el diseño de un servicio elegido por el alumno, utilizando como base el viaje del cliente y DT. Las instrucciones a seguir se suben a PoliformaT, explicando qué tendrán que incluir en cada fase de DT.

Cada curso se han ido revisando las tareas para intentar que cuando los alumnos tienen que hacer la que evalúa, hayan obtenido los conocimientos suficientes para aplicar el método DT para revisar el diseño del servicio que elijan. El primer curso se especificó un servicio concreto, pero desde el segundo curso creímos que era más motivador para ellos que eligieran en base a sus prácticas laborales o intereses. 


\section{Resultados}

En la Tabla 1 se muestran los resultados totales obtenidos en la tarea 2 durante el curso 2018-2019, en la que los alumnos revisaron el diseño del servicio de la biblioteca. Calculando la correlación entre el número de problemas localizados y el número de soluciones, el resultado es $r=0.607$, lo que indica una correlación positiva. Por tanto, aquellos grupos que encuentran más problemas a través de las entrevistas con los usuarios de la biblioteca, también dan más soluciones a dichos problemas.

La correlación entre el número de soluciones y las dificultades consideradas es de $\mathrm{r}=$ 0.418. Por tanto, aquellos grupos que han dado más ideas sobre prosibles soluciones a los problemas, también tendrán más en cuenta qué dificultades deberán considerar a la hora de implementar dichas soluciones.

Tabla 1. Resultados de la tarea 2

\begin{tabular}{|c|c|c|c|c|}
\hline Grupos & $\begin{array}{c}\text { Número de } \\
\text { problemas } \\
\text { que localizan }\end{array}$ & $\begin{array}{c}\text { Número de } \\
\text { soluciones }\end{array}$ & $\begin{array}{c}\text { Consideran } \\
\text { cómo incluir } \\
\text { las ideas }\end{array}$ & $\begin{array}{c}\text { Dificultades a } \\
\text { considerar en la } \\
\text { implementación }\end{array}$ \\
\hline A & 3 & 3 & Sí & 2 \\
\hline B & 3 & 5 & Sí & 3 \\
\hline C & 3 & 3 & Sí & 3 \\
\hline D & 4 & 4 & Sí & 2 \\
\hline E & 3 & 3 & No & 4 \\
\hline F & 2 & 4 & Sí & 3 \\
\hline G & 5 & 6 & Sí & \\
\hline
\end{tabular}

Podríamos, por tanto, concluir que si en este caso hubiéramos indicamos a los grupos un número mínimo de problemas igual o superior a 4 , habrían dado más soluciones cada uno. Dando más soluciones, habrían pensado también en más dificultades a la hora de implementar dichas soluciones.

Este resultado también interesa a la hora de definir la tarea final, ya que solemos decirles que se centren en un número de soluciones concreto sobre las que trabajar. Dado que al no trabajar en equipos multidisciplinares y que el tiempo disponible para hacer la tarea es limitado, llegar a prototipos y testarlos es difícil para los alumnos de esta asignatura. Probablemente, habría que pedirles un esfuerzo mayor en las primeras fases, tratando de que se centren más en obtener necesidades de los usuarios y problemas, analizarlos con más detalle y plantear más ideas innovadoras.

Respecto al cumplimiento de objetivos planteados en la aplicación de DT, la Tabla 2 presenta los porcentajes alcanzados para cada uno de ellos. Como se comprueba en los 
datos de la tabla, los objetivos en los que los alumnos muestran mayor dificultad están relacionados con el diseño de las preguntas para las entrevistas y su adaptación al viaje del cliente, así como en el uso de la información disponible en redes sociales. Respecto al diseño de las preguntas, se ha comprobado que para ellos es difícil la aplicación de metodologías cualitativas de investigación. Tienden a diseñar cuestionarios para encuestas y les resulta más complicado adaptar la entrevista cualitativa a cada etapa del viaje del cliente. Lo mismo ocurre con el uso de información procedente de las redes sociales. Estos resultados nos indican que deberíamos reforzar las explicaciones e incluirlo en la tarea que hacen de prueba, como la que explicamos sobre la Biblioteca.

Tabla 2. Objetivos alcanzados (\% alumnos o grupos)

\begin{tabular}{|c|c|c|}
\hline Objetivo & $\begin{array}{c}\text { Tarea en } \\
\text { aula }\end{array}$ & Tarea final evaluable \\
\hline $\begin{array}{c}\text { Los alumnos trabajan en base a la cultura } \\
\text { de servicio centrada en el usuario }\end{array}$ & $100 \%$ & $100 \%$ \\
\hline $\begin{array}{c}\text { Utilizan el método de DT para revisar un } \\
\text { servicio y plantear mejoras en él }\end{array}$ & $100 \%$ & $100 \%$ \\
\hline $\begin{array}{c}\text { Dichas mejoras están basadas en el viaje } \\
\text { del cliente (customer journey) }\end{array}$ & $\begin{array}{c}\text { No es parte } \\
\text { de la tarea }\end{array}$ & $\begin{array}{c}91 \% \text { definen las etapas } \\
\text { para el servicio concreto } \\
73 \% \text { adaptan la entrevista } \\
\text { al customer journey }\end{array}$ \\
\hline $\begin{array}{c}\text { Aplican metodología cualitativa (entrevista } \\
\text { cualitativa) para obtener información } \\
\text { directamente del cliente y que observen su } \\
\text { comportamiento }\end{array}$ & $100 \%$ & $100 \%$ \\
\hline $\begin{array}{c}\text { Utilizan información en redes sociales que } \\
\text { los clientes hayan escrito sobre el servicio } \\
\text { que se revisa, si está disponible }\end{array}$ & $\begin{array}{c}\text { No es parte } \\
\text { de la tarea }\end{array}$ & \\
\hline
\end{tabular}

Los resultados nos ayudan a entender la importancia del uso de metodologías como el DT, que se apoyen en metodologías cualitativas, para que los alumnos sean capaces de trabajar tanto con metodologías cuantitativas como cualitativas.

El DT permite, además, utilizar otros instrumentos conocidos para diseñar y revisar el diseño de los servicios, como es el viaje del cliente (customer journey). Pero estos instrumentos se centran en buscar mejoras en la experiencia del cliente, mientras que el DT permite dar una visión más completa del diseño de un servicio, desde que se analizan las necesidades del usuario hasta que se consideran los posibles problemas en la implementación de las mejoras. Y al ser un método estructurado, permite también una representación visual del proceso, lo que ayuda a entender mejor las diferentes etapas del método. 


\section{Conclusiones}

En esta comunicación hemos presentado cómo se utiliza DT en una asignatura optativa de la Facultad de ADE. Para ello hemos explicado las diferentes tareas que hacen los alumnos para aplicar DT en la revisión de un servicio.

Además de exponer cada una de las tareas y los objetivos planteados para su diseño, hemos analizado algunos resultados para ver si deberíamos rediseñar alguna de dichas tareas. Los resultados nos dicen que es mejor plantear un número mínimo de problemas y soluciones, así como centrar más las tareas en las tres primeras fases de DT, dadas las características de la titulación y la no posibilidad de trabajar en un entorno multidisciplinar. También que deberíamos reforzar la parte relacionada con las metodologías cualitativas, tanto en el diseño como en el análisis de la información.

Aunque nuestra experiencia en aplicar DT en proyectos reales nos ha mostrado las ventajas de su uso en entornos multidisciplinares. Esta es una limitación en el caso que presentamos. Sin embargo, al ser la mayoría de los alumnos Erasmus, se trabaja en un entorno multicultural, que podría ayudar a plantear un mayor número de ideas innovadoras, ya que los grupos pueden transmitir mejoras procedentes de diseños de los servicios de sus países de origen.

Sería interesante plantearse la posibilidad de trabajar alguna tarea con alumnos de titulaciones de otros centros, de manera que se diera ese entorno multidisciplinar, que sí encontramos en asignaturas de master.

\section{Referencias}

ARMSTRONG, C.E. (2016). "Teaching Innovation Through Empathy: Design Thinking in the Undergraduate Business Classroom”, en Management Teaching Review, 1, 3, p. 164-169.

BROWN, T. (2008). "Design Thinking", en Harvard Business Review, Junio, 84-92.

CHEN, S., BENEDICKTUS, R., KIM, Y. y SHIH, E. (2018). "Teaching Design Thinking in Marketing: Linking Product Design and Marketing Strategy in a Product Development Class", en Journal of Marketing Education, 40, 3, p. 176-187.

D.SCHOOL AT STANFORD UNIVERSITY (2018). D.School Bootleg Deck. $<$ https://dschool.stanford.edu/resources/design-thinking-bootleg> (Consulta: 23 Marzo, 2019).

DE-MIGUEL-MOLINA, B., DE-MIGUEL-MOLINA, M., SEGARRA-OÑA, M. y SANTAMARINA-CAMPOS, V. (2018). "Design Thinking, Business Model Canvas and Intellectual Property Rights. Applying management tools to the AiRT Project". Hedderich et al. (ed.). En Business Meets Technology, Proceeding of the 1st International Conference of the University of Applied Sciences Ansbach. Ansbach (Alemania): Hoschule Ansbach, p. 108-111.

JAARON, A.A.M. y BACKHOUSE, C.J. (2018). "Operationalisation of service innovation: a systems thinking approach", en The Service Industries Journal, 38, 9/10, p. 561-583.

LEWRICK, M., LINK, P. y LEIFER, L. (2018). The Design Thinking Playbook. New Jersey y Canadá: Wiley. 
LIEDTKA, J. (2018). "Why Design Thinking Works", en Harvard Business Review, SeptiembreOctubre, p. 72-79.

SANTAMARINA-CAMPOS, V., DE-MIGUEL-MOLINA, M., DE-MIGUEL-MOLINA, B. y CARABAL-MONTAGUD, M.A. (2018). “Application of Design Thinking for Technology Transfer of Remotely Piloted Aircraft Systems for the Creative Industry", en World Academy of Science, Engineering and Technology. International Journal of Mechanical and Mechatronics Engineering, $12,5,457-463$.

SCHUMACHER, T. y MAYER, S. (2018). "Preparing Managers for Turbulent Contexts: Teaching the Principles of Design Thinking”, en Journal of Management Education, 42, 4, p. 496-523.

ZIDULKA, A. y KAJZER-MITCHELL, I. (2018). "Creativity or Cooptation? Thinking Beyond Instrumentalism When Teaching Design Thinking”, en Journal of Management Education, 42, 6, p. 749-760. 